\title{
Study of infectious conjunctivitis among children in rural areas of Qinghai province
}

\author{
Qingfeng Liang ${ }^{1}$, Xinxin Lu ${ }^{1}$, Mei Wang ${ }^{1}$, Lei Tian ${ }^{1}$, Antoine Labbé ${ }^{1,2,3}$ \& Ailian $\mathrm{Hu}^{1 *}$ \\ ${ }^{1}$ Beijing Institute of Ophthalmology, Beijing Tongren Eye Center, Beijing Tongren Hospital, Capital Medical University, \\ Beijing Key Laboratory of Ophthalmology and Visual Sciences, Beijing 100005, China; \\ ${ }^{2}$ Quinze-Vingts National Ophthalmology Hospital, Paris and Versailles Saint-Quentin-en-Yvelines University, Versailles F-75002 France; \\ ${ }^{3}$ INSERM, U968; UPMC Univ Paris 06, UMR_S 968, Institut de la Vision; CNRS, UMR_7210, Paris F-75012, France
}

Received April 1, 2016; accepted April 8, 2016

\begin{abstract}
Infectious conjunctivitis is no longer a common eye disease in cities and developed areas, but in remote mountainous regions of China where living conditions are relatively under-developed, the occurrence of infectious conjunctivitis remains a public health issue. Here, we evaluate the ratio of infectious conjunctivitis among primary school students in the rural areas of Xunhua, Huangyuan, and Huzhu Counties in the Qinghai province of China, and report the microorganisms involved in causing infection. Of the 765 eligible primary school students, 694 participated to this study (a response rateof 90.7\%), 381 boys and 313 girls, with a mean age of 7.52 years (range 7-9 years). A clinical diagnosis of infectious conjunctivitis was returned for 238 of the 694 children examined. The ratio of infectious conjunctivitis in Xunhua County (46.64\%) was higher compared to that in Huangyuan $(26.67 \% ; P=0.03)$ and Huzhu Counties $(23.61 \% ; P=0.02)$. The leading cause of conjunctivitis was bacterial, followed by chlamydial and viral. Our results show that there is a high ratio of infectious conjunctivitis among rural primary school students in Qinghai province.
\end{abstract}

conjunctivitis, rural children, bacteria, chlamydia

Citation: Liang, Q., Lu, X., Wang, M., Tian, L., Labbé, A., and Hu, A. (2016). Ratio of infectious conjunctivitis among children in rural areas of Qinghai province. Sci China Life Sci 59, 548-554. doi: 10.1007/s11427-016-5058-x

\section{INTRODUCTION}

Conjunctivitis is a common ocular surface disease that is often divided into two types: infectious and non-infectious (Xu and Huang, 2002). Infectious conjunctivitisparticularly bacterial and chlamydial-is no longer common in cities and developed areas (Azari and Barney, 2013). In the remote mountainous regions of China where living conditions are relatively under-developed, infectious conjunctivitis remains a public health issue (Zhang et al., 2014). Children in crowded schools are particularly vulnerable to the spread of this disease and so the initiation of appropriate

*Corresponding author (email: halzxf@sina.com) public health measures relies on knowledge of the ratio of infectious conjunctivitis in this population. The objective of this study was to evaluate the ratio of infectious conjunctivitis in the mountainous rural areas of the Qinghai province and to determine the microorganisms responsible for infection.

\section{RESULTS}

Of the 765 students registered in the selected primary schools, and therefore eligible to participate, 694 were examined for a response rate of $90.7 \%$. 71 students were excluded due to a lack of informed consent or time to participate because of schoolwork. Boys made up 54.89\% of participants $(n=381)$, while $45.11 \%$ were girls $(n=313)$. The 
mean age of participants was 7.52 years with a range of 7-9 years. Only $68(8.93 \%)$ students' visual acuity was less than 1.0

Among 694 students, infection conjunctivitis was diagnosed in $238(34.3 \%)$ of the study subjects; the infection ratio was $46.6 \%$ (139 cases) in Xunhua County, significantly higher than that in Huangyuan county at $26.7 \%$ (48 cases) and in Huzhu County at $23.6 \%$ (51 cases) $\left(\chi^{2}=4.142\right.$, $P=0.03$ and $\chi^{2}=6.198, P=0.02$, respectively). Among those students with infectious conjunctivitis, bacterial conjunctivitis was diagnosed in 213 cases, chlamydial conjunctivitis was seen in 24 cases, and viral conjunctivitis in one case. 24 cases were clinically diagnosed as active trachoma, with 20 cases in the trachoma follicular (TF) and four in the trachoma intense (TI).

Examples of conjunctivitis are shown in Figure 1. Bacterial conjunctivitis (Figure 1A) was bilateral in 196 of the 213 diagnosed cases. Only one child had adenoviral conjunctivitis (Figure 1B), and this case was bilateral with an enlarged pre-auricular gland. All cases diagnosed as trachoma, the TF (Figure 1C) or (Figure 1D), were bilateral. Corneal involvement and neovascularization were not detected in any child.

Specimens were collected for bacterial culture from binocular conjunctival cul-de-sacs of the 213 students diagnosed with bacterial conjunctivitis. Of these, 153 cases (72\%) had a positive result for bacterial culture. A total of 251 bacterial strains were isolated: 114 (45.42\%) Gram-positive cocci, $26(10.36 \%)$ Gram-positive bacilli, 3 (1.20\%) Gram-negative cocci, and 108 (43.02\%) Gramnegative bacilli. The most frequently isolated bacteria were Corynebacterium macginleyi (21.5\%), coagulase-negative Staphylococcus (21\%), Moraxella bacillus (12\%), Microbacterium (10\%) and Haemophilus influenzae (8.7\%) (Тa- ble 1). In the 213 patients with bacterial conjunctivitis, two strains of different bacteria were isolated in 53 patients, and three strains were foundin 13 cases.

The 88 most frequent Gram-positive strains isolated from patients with bacterial conjunctivitis, as well as 55 strains of Gram-negative bacteria were used for antimicrobial sensitivity testing (Figure 2). Sensitivity to levofloxacin, tobramycin, azithromycin, and ampicillin was 98.3\%, 82.9\%, $72.9 \%$, and $71.5 \%$, respectively, for Gram-positive bacteria, and $85.7 \%, 78.5 \%, 76.9 \%$, and $64.0 \%$, respectively for Gram-negative bacteria.

Ofthe 24 patients with a clinical diagnosis of chlamydial conjunctivitis, 22 cases (92\%) were positive for the Chlamydia trachomatis PCR test. The TI phase had a higher positivity rate $(99.5 \%)$ than the TF $(75.0 \%)$, but this was not statistically significant $\left(\chi^{2}=2.125, P=2.125\right)$. Of these 22 positive cases, $18(82 \%)$ were also positive for bacterial culture, which detected $H$. influenzae, C. macginleyi, and coagulase-negative Staphylococcus. At least two strains of bacteria were identified in 11 chlamydial conjunctivitis cases.

An adenovirus multiplex nested PCR test was conducted in all of the 213 conjunctivitis specimens. One case diagnosed as adenoviral conjunctivitis returned a positive result in both eyes.

\section{DISCUSSION}

Conjunctivitis is the most common ocular surface disease, especially in children (Dominique et al., 2010). There are more than 6 million patients diagnosed with acute conjunctivitis in the United States each year, with the cost of treating bacterial conjunctivitis alone estimated at \$380-860 million per year (Smith and Waycaster, 2009; Udeh et al.,

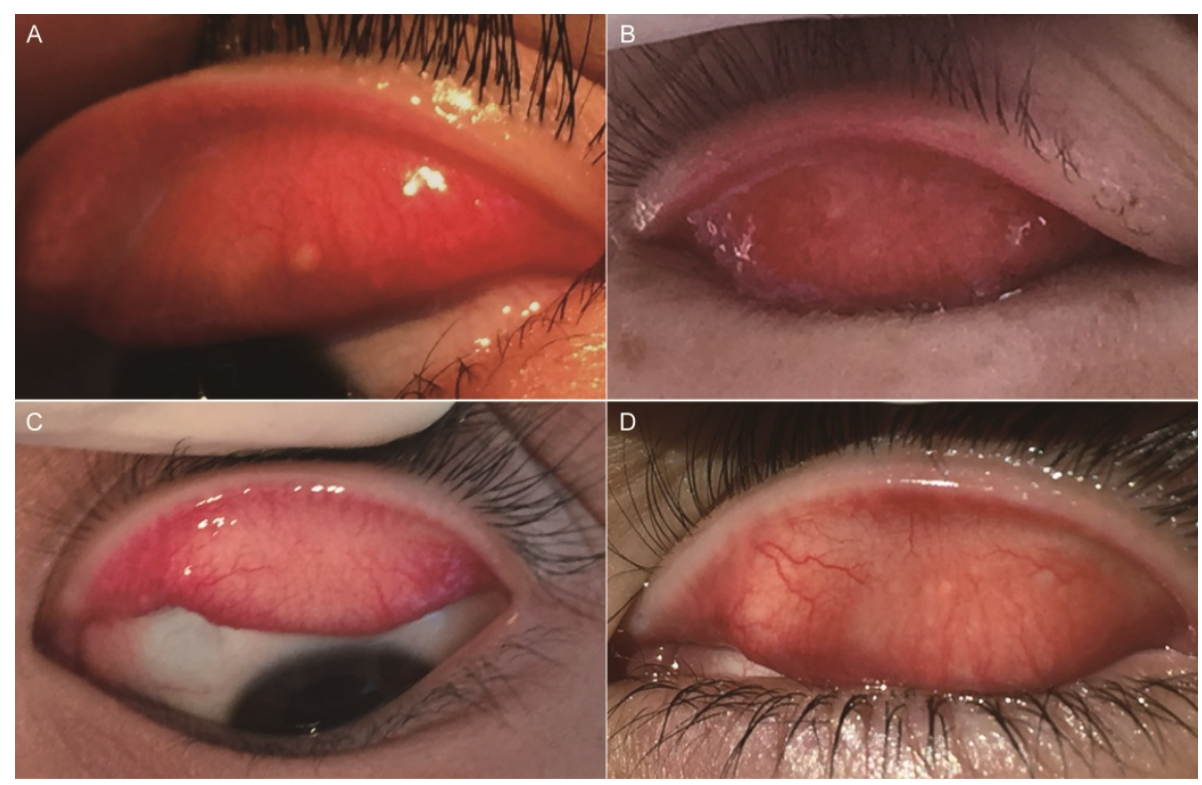

Figure 1 Images of infectious conjunctivitis. A, Bacterial conjunctivitis. B, Adenoviral conjunctivitis. C, TF. D, TF and TI. 
Table 1 Distribution of pathogenic isolates in patients with bacterial conjunctivitis

\begin{tabular}{|c|c|c|}
\hline Name of the bacteria & Number of strains & Percentage of the total bacteria (\%) \\
\hline Gram-positive cocci & 114 & 45.42 \\
\hline Coagulase-negative Staphylococcus & 53 & 21.12 \\
\hline Streptococcus pneumoniae & 20 & 7.97 \\
\hline Staphylococcus aureus & 15 & 5.98 \\
\hline Micrococcus & 12 & 4.78 \\
\hline Streptococcus mitis & 5 & 1.99 \\
\hline Streptococcus agalactiae & 4 & 1.59 \\
\hline Gram-positive bacilli & 26 & 10.36 \\
\hline Microbacterium & 26 & 10.36 \\
\hline Gram-negative bacilli & 108 & 43.02 \\
\hline Corynebacteriummacginleyi & 54 & 21.51 \\
\hline Moraxella bacillus & 32 & 12.75 \\
\hline Haemophilusinfluenzae & 22 & 8.76 \\
\hline Moraxella catarrhalis & 2 & 0.80 \\
\hline Neisseria meningitidis & 1 & 0.40 \\
\hline
\end{tabular}

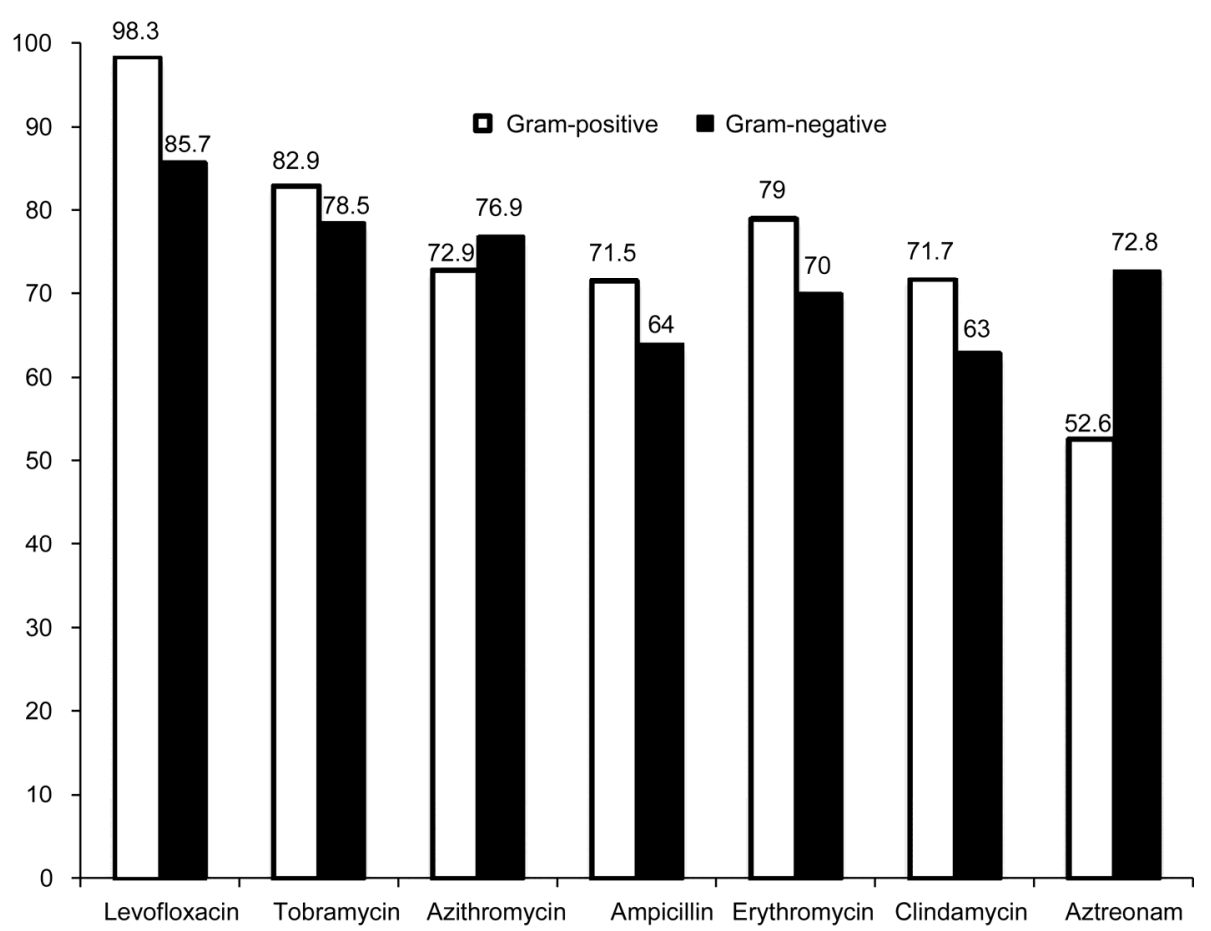

Figure 2 Drug sensitivity of isolated pathogens to common antibiotics.

2008). Many US state health departments require students to be treated with topical antibiotic eye drops before returning to schoolirrespective of the underlying cause of conjunctivitis (Azari and Barney, 2013). Industrialized countries place great importance on children's infectious conjunctivitis. With the development of the Chinese economy, infectious diseases-including infectious conjunctivitis-are no longer major public health problems, but monitoring the ratio of infectious conjunctivitis remains paramount in rural or remote mountainous areas lacking water and medical supplies (Wei, 2011). Therefore, this study focused on the ratio of infectious conjunctivitis in children in the rural mountainous regions of the Qinghai province, and the distribution of the responsible microorganisms.

In rural areas of the Qinghai province, the ratio of infectious conjunctivitis in children was $34 \%$, significantly higher than that observed in American children (13.5\%) (Ohnsman, 2007), and also higher than the rate in pre-school aged students in the rural and suburban areas of Beijing (22.59\%), reported in a study of 50,000 students (Xiang et al., 1997). The higher ratio in this region is probably associated with poor hygienic practices and local med- 
ical conditions. Although we cannot get detailed data about water resources in this remote area, we found that students did not have sufficient amounts of clean water to wash their faces or hands, and the faces and hands of $80 \%$ of the students were dirty. Our findings indicate that infectious conjunctivitis is still a frequently occurring infection in Chinese children, particularly in rural areas, but even in urban locations. It is necessary for medical practitioners and teachers to educate students and their parents about simple prevention steps, such as proper hand washing with flowing water, and that medical treatment should be sought quickly if conjunctivitis is suspected.

Our data showed that bacterial infection was the most frequent form of infectious conjunctivitis, consistent with the findings of Alfonso et al (Alfonso et al., 2015). The common isolates in this study, C. macginleyi and coagulase-negative Staphylococcus, differed from those isolated in the United States (H. influenza and Streptococcus pneumonia) (Mah, 2006). Coagulase-negative Staphylococci are the most frequent microorganisms causing infectious conjunctivitis in children, followed by coryneform bacteria and entero bacteria (Cao et al., 2010). This may be due to external environmental conditions, and more opportunities for the conjunctival sacs of human eyes to become infected by these bacteria. Most of the isolated bacteria were sensitive to common antibiotics (levofloxacin, tobramycin, and azithromycin).

In this study, one case of adenoviral conjunctivitis was diagnosed. Hospital-based investigations of acute conjunctivitis showed that viral conjunctivitis was highly prevalent, accounting for $80 \%$ of acute conjunctivitis cases in the United States (Kaufman, 2011). However, in this schoolbased survey, this trend was not seen because students generally had medical treatment for acute onset. The student diagnosed with adenoviral conjunctivitis had not been seen by an ophthalmologist and maintained close contact with his classmates. It is therefore of utmost importance to provide health education with regards to personal hygiene as well as the prevention and treatment of infectious diseases in rural areas.

Trachoma is a blinding eye disease caused by $C$. trachomatis infection. Before the liberation of China, the averageratio of trachoma was about $55 \%$, and as high as $80 \%-90 \%$ in remote rural areas (Wang et al., 2015). Following decades of effort, in the previous epidemiological investigation, TF among primary school students in Qinghai province was less than $5 \%$, but there were still some sporadic cases. Our study found several trachoma students without corneal complications, all of cases with TF or TI. Reaching the goal of eliminating blinding trachoma does not mean that China has no trachoma cases (Wang and $\mathrm{Hu}$, 2015). The results of our study required our continuing intervention promoted by the surgery, antibiotics, facial cleanliness, and environmental improvements (SAFE strategy).
Three counties representing different economic and health statuses were selected using a stratified sampling method. Huangyuan County, located in the southeast of Qinghai Lake, isat the boundary between the agricultural and pastoral areas of Qinghai, with better economic conditions and school buildings, as well as plenty of drinking and washing water for students. The primary school of Galeng village in Xunhua County is located at an elevation of more than 3,000 meters and surrounded by mountains, lacking water and education in good hygiene practices. The ratio of infectious conjunctivitis in Xunhua County was significantly higher than that in Huangyuan County and Huzhu County, so it is imperative to provide a clean water supply, develop good hygiene practices, and generally strengthen health consciousness, so as to prevent the onset of infectious conjunctivitis (Yang et al., 2013).

In conclusion, infectious conjunctivitis for children attending school in remote rural mountainous areas is still a major public health concern. This data should encourage local medical institutions to take appropriate preventative measures to control infectious conjunctivitis. We recommend that educational and health institutions provide some instructions on infectious conjunctivitis and using good hygienic practices to children in underserved areas. This will not only have an effect on infectious conjunctivitis, but will also likely positively impact general health as well.

\section{MATERIALS AND METHODS}

\section{Sample size}

The reported ratio of infectious conjunctivitis (24.6\%) in children attending school in the city of Changzhou (Hebei province) was used to calculate the sample size (Yang et al., 2014) using the formula $n=Z p(1-p) / B^{2}$, where $Z$ is 1.96 for $95 \%$ confidence level, $p$ is the percentage of infectious conjunctivitis ratio (24.6\%) and $B$ is the confidence interval (Stokes and Allor, 2016). An appropriate sample size of 760 was obtained.

\section{Selectionof province and schools}

The Qinghai province includes a prefecture-level city (Xining city), one region (Haidong region), and six ethnic autonomous prefectures consisting of 37 counties. We created a stratified random sample using four steps: (i) Counties were selected based on different levels of economic and cultural development (high level: Huangyuan County; ordinary: Huzhu County; and low level: Xunhua County). (ii) In each county, two elementary schools were randomly selected with consecutive numbers. (iii) We listed all of the students aged 7-12 years in the target school; and (iv) Calculated the proportionate stratification. Samples involved 324, 199, and 242 cases from Xunhua County, Huangyuan County, and Huzhu County, respectively, for a total of 765 subjects. 


\section{Clinical examination and microbiological test}

This study was performed from May to December of 2015, and was approved by the ethics committee of Beijing Tongren Hospital, Capital Medical University (No. TRKY032). All students had resided in the Qinghai region for more than six months. The parents or legal representatives of the students were informed ofthe purpose of this study and provided informed consent, and assisted the studentsin providing demographic information and medical history. Students having used antibiotic eye drops for up to $3 \mathrm{~d}$ prior to the investigation were excluded from the study. The investigation included two phases: on-site inspection (clinical examination and specimen sampling) and laboratory examination (Figure 3).

The diagnosis of infectious conjunctivitis and subsequent sample collection were performed by qualified medical professionals from Beijing Tongren Hospital, who were trained in the diagnosis and laboratory investigation of conjunctivitis by specialists from World Health Organization (WHO).

Clinical examination included eliciting the subjective symptoms of conjunctivitis (foreign body sensation, photophobia, red eyes, excessive tearing, and discharge). This was followed by standardized visual acuity testing and slit lamp examination of the eyelids, conjunctiva, and cornea. The presence of discharge, vascular congestion, edema, size and number of papillae and follicles, and presence of palpebral conjunctiva scarring were evaluated and recorded. The cornea was examined for transparency, neovascularization, and the presence of infiltrates and edema.
In patients with a clinical diagnosis of conjunctivitis, samples for bacterial isolation were obtained from the upper palpebral conjunctiva and lower conjunctival sac of both eyes. The four samples obtained from each child were contained separately during transportation. The specimens collected from the upper palpebral conjunctiva were used for C. trachomatis PCR while those from the lower conjunctival sac were used for bacterial culture and adenovirus multiplex nested PCR detection.

\section{Diagnostic criteria}

The diagnosis of bacterial conjunctivitis was based on: (i) unilateral or bilateral palpebral or bulbar conjunctival congestion or edema; (i) mucus or mucopurulent discharge in the involved eye; (ii) positive bacterial culture (Gong et al., 2010). Both criteria (i) and (ii) were required to make a clinical diagnosis of conjunctivitis.

The diagnosis of trachoma based on WHO recommendations is shown in Table 2; the five major signs issued by WHO in 1987 were used as diagnostic and grading criteria for trachoma in this study (Wang and $\mathrm{Hu}, 2014$ ).

The diagnosis of viral conjunctivitis was based on: (i) an acute onset disease with pain and/or discomfort in the eye, photophobia, tearing usually involving one eye at first and then the second 3-5 d later. (ii) Palpebral conjunctiva congestion, edema, follicular hyperplasia, and subconjunctival hemorrhage if present; (iii) Serous secretion and (iv) pre-auricular lymph node enlargement. Criteria i, ii and iii were all required to make the diagnosis (Azari and Barney, 2013).

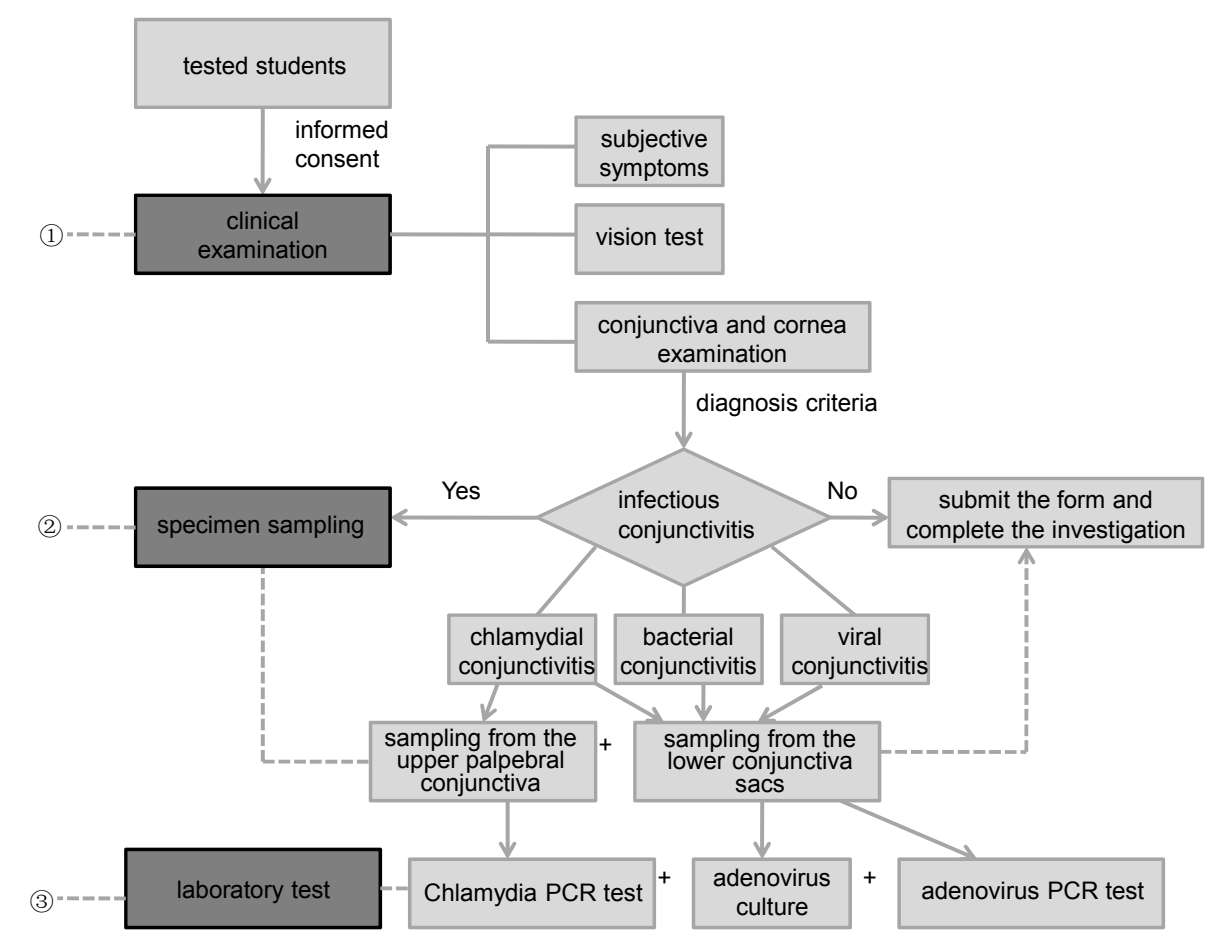

Figure 3 Epidemiological investigation of infectious conjunctivitis among children in rural areas of the Qinghai province. 
Table 2 Simplified WHO trachoma grading system

\begin{tabular}{ll}
\hline Grade & Clinical signs \\
\hline Trachomatous inflammation follicles (TF) & Five or more follicles of $>0.5$ mm on the upper tarsal conjunctiva \\
Trachomatous inflammation intense (TI) & Inflammatory thickening obscuring more than half the normal deep tarsal vessels \\
Trachomatous conjunctival scarring (TS) & The presence of easily visible scars in the tarsal conjunctiva \\
Trachomatous trichiasis (TT) & At least one eyelash rubbing on the eyeball or evidence of recent removal of in-turned eyelashes \\
Cornea opacity (CO) & Opacity of the cornea involving part of the pupil margin \\
\hline
\end{tabular}

\section{Laboratory testing}

The isolation and identification of bacteria were carried out according to the national guide to clinical laboratory procedures (Yan et al., 2009) and ATB identification strips provided by BioMerieux (France) were used for identification of strains. Etest was employed as drug sensitivity test for the major pathogenic bacteria obtained on culture. The antibiotics used for the drug sensitivity test included levofloxacin, tobramycin, azithromycin, ampicillin, erythromycin, clindamycin, and aztreonam (Yan et al., 2009).

Reagent kits (genomic DNA from tissue kits, Macherey-Nagel, Germany) were used to extract the DNA of C. trachomatis. DNA from the C. trachomatis endogenous plasmid was PCR-amplified using primers designed from those referenced in the literature (Kapil et al., 2015).

DNAZOL reagent kits (Invitrogen, USA) were used to extract adenovirus DNA from conjunctivitis specimens and standard strains. $20 \mu \mathrm{L}$ of $\mathrm{NaOH}\left(8 \mathrm{mmol} \mathrm{L}^{-1}\right)$ was used to dissolve DNA, which was then amplified twice by multiplex nested PCR, and the bands of amplified DNA were observed under a UV lamp (Deng et al., 2007; Morozumi et al., 2014).

\section{Statistical methods}

SPSS 17.0 (SPSS Inc., USA) was used for statistical analysis. The $\chi^{2}$ test was used for comparison of proportions, and single factor and multi-factor analysis of variance (ANOVA) were used to analyze the measurement data. $P<0.05$ was considered as statistically significant.

Compliance and ethics The author(s) declare that they have no conflict of interest.

Acknowledgements This work was supported by National Natural Science Foundation of China (81470607).

Alfonso, S.A., Fawley, J.D., and Lu, X. (2015). Conjunctivitis. Prim Care $42,325-345$.

Azari, A.A., and Barney, N.P. (2013). Conjunctivitis: a systematic review of diagnosis and treatment. JAMA 310, 1721-1729.

Cao, S., Pei, B., and Li, Y. (2010). Analysis on drug resistance of the pathogenic bacteria isolated from secretions of the eyes of 715 children. Chin Med Guide 8, 31-32.

Deng, J., Qian, Y., and Zhao, L. (2007). Application of multiplex nested
PCR in the detection and classification of Adenovirus. Chin J Epidemiol 28, 781-784.

Dominique, B.G., Patricia, M.K., Ariel, B., Lamia, E.F., Yasmin, B., Pascale, P., Laurent, D., Pablo, G., Edouard, B., and Isabelle, C. (2010). Efficacy and safety of azithromycin $1.5 \%$ eye drops for purulent bacterial conjunctivitis in pediatric patients. Pediatr Infect Dis J 29, 222-226.

Gong, L., Sun, X., Qiu, X., Zhang, Y., Qu, J., Yuan, Z.L., and Xiong, Q.C. (2010). Comparative research of the efficacy of the gatifloxacin and levofloxacin for bacterial conjunctivitis in human eyes. Chin J Ophthalmol 46, 525-531.

$\mathrm{Hu}, \mathrm{A}$. , Cai, X., and Li, Y. (2015). Comparison on vision disability caused by trachoma between 1987 and 2006. Chin J Ophthalmol 51, 484-486.

Kaufman, H.E. (2011). Adenovirus advances: new diagnostic and therapeutic options. Curr Opin Ophthalmol 22, 290-293.

Mah, F. (2006). Bacterial conjunctivitis. Clin Evid 2012, 756-761.

Morozumi, M., Shimizu, H., Matsushima, Y., Mitamura, K., Tajima, T., Iwata, S., and Ubukata, K. (2014). Evaluation of new immunochromatographic assay kit for adenovirus detection in throat swab: comparison with culture and real-time PCR results. J Infect Chemother 20, 303-306.

Ohnsman, C.M. (2007). Exclusion of students with conjunctivitis from school: policies of state departments of health. J Pediatr Ophthalmol Strabismus 44, 101-105.

Kapil, R., Press, C.G., Hwang, M.L., Brown, L., and Geisler, W.M. (2015). Investigating the epidemiology of repeat Chlamydia trachomatis detection after treatment by using $C$. trachomatis OmpA genotyping. J Clin Microbiol 53, 546-549.

Smith, A.F., and Waycaster, C. (2009). Estimate of the direct and indirect annual cost of bacterial conjunctivitis in the United States. BMC Ophthalmol 9, 1-11.

Stokes, L., and Allor, J.H. (2016). A power analysis for fidelity measurement sample size determination. Psychol Methods 21, 35-46.

Udeh, B.L., Schneider, J.E., and Ohsfeldt, R.L. (2008). Cost effectiveness of a point-of-care test for adenoviral conjunctivitis. Am J Med Sci 336, 254-264.

Wang, N., and Hu, A. (2015). Enlightenment and thinking of blinding trachoma elimination in China. J Am Med Assoc 51, 1022-1022.

Wang, N., and Hu, A. (2014). Blindness Prevention Handbook. (Beijing: People's Medical Publishing House), pp. 52-71.

Wang, N., Hu, A., and Taylor, H.R. (2015). Trachoma. (Beijing: People's Medical Publishing House), pp. 45-46.

Wei, L. (2011). Study on hazardous behaviors to health among adolescent and the progress of intervention measures. Chin J Public Health 27, 933-934.

$\mathrm{Xu}$, W., and Huang, H. (2002). Analysis of bacterial culture results in ocular infections. Ophthalmol China 2, 78-79

Xiang, L., Shen, F., and Jiang, A. (1997). Investigation report on eye diseases among 50,000 children in suburb areas of Beijing. Chin J Strabismus Pediatric Ophthalmol 5, 23-25.

Yan, Y., Wang, Y., and Shen, Z. (2009). National health and family planning commission of the people's republic of China: National clinical laboratory procedures, pp. 720-724.

Yang, L., Zhao, Y., and Yang, Y. (2014). Study on the eye diseases among school age children of 6-14 years old in the urban areas of cangzhou 
city, Hebei province. Int J Ophthalmol 14, 908-912.

Yang, S., Pan, X., Wang, H., and Zhao, G. (2013). A randomized, double-blind and placebo-controlled clinical trail of topical administration of $1 \%$ azithromycin eye drops for acute bacterial conjunctivitis. Chin J
Exp Ophthalmol 31, 182-185.

Zhang, R., Mao, X., and Yu, Y. (2014). Reseach of Dongzhi yuan area of eye surface bacterial inflammation pathogens and antibiotic application. Foreign Med Sci Sect Medgeogr 35, 139-141.

Open Access This article is distributed under the terms of the Creative Commons Attribution License which permits any use, distribution, and reproduction in any medium, provided the original author(s) and source are credited. 